\title{
Tolerability, Efficacy and Feasibility of Concurrent Gemcitabine and Cisplatin (CGP) Combined With Intensity Modulated Radiotherapy for Loco-Regionally Advanced Carcinoma of the Cervix
}

Emmanuel Kwateng Drokow ${ }^{1}$, Liu Zi² ${ }^{2}$ Han Qian ${ }^{1}$, Lanlan $\mathrm{Xu}^{1}{ }^{1}$, Francis Foli ${ }^{3}$, Hafiz Abdul Waqas Ahmed ${ }^{4}$ Gloria Selorm Akpabla ${ }^{5}$, Guangyin $\mathrm{Wu}^{1}$, Emmanuel Bamfo Agyekum${ }^{6}$, Weihua Gao', Marie-Anne Deku ${ }^{7}$, Juanjuan Song ${ }^{4}$, Kai Sun ${ }^{\bowtie}$

1. Department of Radiation Oncology, Zhengzhou University People's Hospital \& Henan Provincial People's Hospital 450003, China

2. Department of Radiation Oncology, First Affiliated Hospital of Xi'an Jiaotong University, Xi' an 710061, China

3. Department of Internal Medicine, Seventh Day Adventist Hospital, Takoradi MC 1034, Ghana

4. Department of Haematology, Zhengzhou University People's Hospital \& Henan Provincial People's Hospital 450003, China

5. Department of Internal Medicine, Tianjin Medical University, Tianjin 300033, China

6. Department of Pharmacy, Zhengzhou University, 450001, Zhengzhou-China.

7. Department of Oncology, Binzhou Medical University, Yantai-Shandong 264003, China

$\square$ Corresponding author: Kai Sun, MD, PhD., Department of Haematology, Zhengzhou University People's Hospital \& Henan Provincial People's Hospital 450003, China. Email: sunkai@cellscience.org

(C) The author(s). This is an open access article distributed under the terms of the Creative Commons Attribution License (https://creativecommons.org/licenses/by/4.0/). See http://ivyspring.com/terms for full terms and conditions.

Received: 2019.09.14; Accepted: 2020.01.20; Published: 2020.02.19

\begin{abstract}
Background: Gemcitabine and cisplatin combined with conventional radiotherapy in treating patients with cervical cancer, resulted in a favourable conclusion but accompanied with high toxicity. The objective of our research was to assess the tolerability, efficacy and feasibility of dual chemotherapy in addition to image-guided adaptive brachytherapy and highly conformal external beam radiation therapy.

Methods \& Materials: From June 2011 to November 2013, 81 cervical cancer patients with FIGO stage IB2IIIB medical records were retrospectively reviewed. All patients received whole pelvic radiotherapy (WPRT) to a total dose of $50.4 \mathrm{~Gy} / 1.8 \mathrm{~Gy}$ Chemoradiotherapy prescription objectives were: concurrent gemcitabine (125 $\left.\mathrm{mg} / \mathrm{m}^{2}\right)$ and cisplatin $\left(30 \mathrm{mg} / \mathrm{m}^{2}\right)$ during the 6 weeks of external beam radiation therapy (EBRT) followed by two cycles of gemcitabine $\left(1 \mathrm{~g} / \mathrm{m}^{2}, \mathrm{dl}, \mathrm{d} 8\right)$ and cisplatin $\left(25 \mathrm{mg} / \mathrm{m}^{2} \mathrm{dl}-\mathrm{d} 3\right)$ on the tenth week. External beam radiotherapy was followed by image-guided brachytherapy of $24 \mathrm{~Gy} / 4$ fractions. Version 4 of the common terminology criteria for adverse events (CTCAE v 4.0) was used in grading the toxicities.

Results: Sixty-nine patients obtained complete response (CR), six had a partial response (PR), and five patients had stable disease (SD). The disease control rate $(D C R=S D$ and $O R R)$ and overall response rate $(O R R=P R$, CR or PR) were $92.6 \%$ and $85.2 \%$ respectively. The 3-year and 5-year estimated overall survival (OS) was $75.4 \%$ and $66.3 \%$, and the 3-year and 5-year estimated progression-free survival (PFS) were $78.2 \%$ and $65.4 \%$. The median PFS time and OS time were 36.8 months and 45.5 months, respectively. Distance metastasis was evident in the lung ( 3 patients), pelvic wall ( 2 patients), liver ( 3 patients) and bone ( 2 patients). Six (6) had a local relapse, and two (2) patients had local relapse plus simultaneous systemic metastatic tumour.

Conclusions: Unlike past results, gemcitabine and cisplatin appear to be tolerable, efficient and feasible when combined with conformal radiotherapy.
\end{abstract}

Key words: gemcitabine, cisplatin, cervical cancer, chemoradiotherapy, overall survival

\section{Introduction}

As the second most frequently diagnosed cancer and the third major cause of death in female, cervical cancer is estimated to have a prevalence and mortality rate of 527,000 and 265,700 respectively, representing 
$8 \%$ of total cancer death and cases in females [1,2]. Squamous cell carcinoma (80-90\%) and adenocarcinoma are the common histology type seen at diagnosis [3-5]. Presently, the standard treatment and management of cervical cancer are chemoradiotherapy, surgery or high dose intracavitary brachytherapy. Numerous prospective randomized control studies have demonstrated that combined cisplatin-based chemoradiotherapy reduces death rates, increases and improves disease-free survival. Furthermore, substantial evidence revealed that patients treated with cisplatin-based chemoradiotherapy have a better outcome than those who received only radiation therapy, even though it is correlated with higher side effects. The combination of this multimodal approach results in an abject $12 \%$ increase in 5-year overall survival compared to radiation alone [6].

The open phase III clinical trial conducted by Duenas et al evaluated the therapeutic advantages of chemoradiotherapy, and the results laid the basis for concomitant chemoradiotherapy (CCRT) to be the preferred therapy for loco-regionally advanced carcinoma of the cervix [7]. Intensity-modulated radiotherapy (IMRT) has altered the prognosis for cervical cancer with the development of medicine and contemporary delivery techniques in radiation treatment. The inclusion of multimodal imaging, particularly in cervical cancer patients, improves systemic and or nodal tumour detection while enabling the better description of loco-regionally tumour and selection of patients. Using dose-escalated radiation therapy, it is now feasible to treat metastatic lymph nodes without serious toxicity. Notwithstanding all the progress, however, systemic disease recurrence continues to be an issue after treatment. The challenge of decreasing the recurrence of diseases is well recognized, and the future response may be to add another chemotherapeutic drug to the present accepted treatment of single-agent cisplatin. A few trials revealed promising outcomes with chemotherapies involving cisplatin and anthracycline. However, since anthracyclines are linked with serious and severe cardiac toxicity and bone-marrow suppression, less toxic non-anthracycline regimens need to be investigated.

Gonźales et al reported there was a significant reduction in toxicity when gemcitabine and cisplatin were used in advanced cervical cancer patients. The synergy between gemcitabine and cisplatin has been demonstrated by multiple preclinical and clinical trials [7]. With that in mind, we chose the cisplatin and gemcitabine (GP) combination for loco-regionally advanced carcinoma of the cervix (LRACC) in our institution, since the mechanism of action of gemcitabine varies from previous agents. Furthermore, this combined regimen has demonstrated small toxic profile. Additionally, using gemcitabine plus cisplatin in treating other squamous cell carcinomas has shown promising effectiveness [8-11].

Gemcitabine is a deoxycytidine analogue and wide-spectrum antimetabolite with antineoplastic activity. The antineoplastic nature of gemcitabine has resulted in its usage in several forms of advanced cancers. Gemcitabine is a drug with single-agent action in recurrent or metastatic cervical carcinoma but has demonstrated clear radio-sensitizing characteristics in preclinical studies, even in human cervical cell lines.

The objective of our research was to evaluate the tolerability, efficacy and feasibility of dual chemotherapy using gemcitabine and cisplatin regime in addition to image-guided adaptive brachytherapy and highly conformal external beam radiation therapy in loco-regionally advanced carcinoma of the cervix.

\section{Method \& Materials}

\section{Patients}

Histologically confirmed carcinoma of the cervix patients with FIGO stage 1B2-IIIB tumours treated with concurrent gemcitabine plus cisplatin and IMRT at the Radiation Oncology Department of Zhengzhou University People's Hospital were involved in our research. The study was approved by the institutional review and ethics board of Zhengzhou University. The pre-treatment histories, medical and follow-up records of 81 treated patients from June 2011 to November 2013 were reviewed retrospectively. The 2009 International Federation of Gynecology and Obstetrics staging system were used.

\section{Pre-treatment workup}

All the patients included in our study underwent our institutional pre-treatment workup protocol (reviewing previous medical records, gynaecological and general physical examination, tumour biopsy, digital rectal examination (DRE), complete blood count ( $\mathrm{CBC})$, biochemical examinations, cystoscopy and rectoscopy). The eligibility criteria included:

- Histologically confirmed carcinoma of the cervix patients

- Patients without a previous history of being treated for any type of cancer

- KPS score above 70

- No evidence of distance metastasis

- Patients with normal liver functions

The exclusion criteria included patients with evidence of distance metastasis, benign tumours, and cervical sarcoma patients. 


\section{Radiotherapy Planning}

Imported into the pinnacle treatment planning system (TPS) were images obtained by diagnostic 18FDG-PET/CT, CT and MRI. The RTOG guidelines were used in contouring. Based on all accessible medical and imaging information, the gross tumour volume (GTVc) was described as the visible macroscopic tumour. The clinical target volume (CTV) involved the regional lymph nodes and primary tumour sites. The primary clinical target volume encompassed gross tumour volume, parametrium, cervix, uterus and upper one-third of the vagina. In instances, where there was involvement of the vagina, the clinical target volume was extended $20 \mathrm{~mm}$ into the tumour's vagina caudal. The planning target volume (PTV) was generated using an isotropic expansion with the internal target volume (motion of uterus, cervix and surrounding organs) taken into account. The planning target volume was expanded $1 \mathrm{~cm}$ laterally and $1.5 \mathrm{~cm}$ in the anterior-dorsal direction. Correction of the planning volume was done when necessary [12-14].

The PTV was prescribed to a total dose of 50.4 Gy. Tumour response assessment using magnetic resonance imaging was done after patients had received 45Gy of the total dose. In situations where the rest of the tumour was bigger than four $\mathrm{cm}$ in diameter, the PTV received an additional 9 Gy boost while tumours less than four $\mathrm{cm}$ after evaluation also received an additional 5.4 Gy boost. The patient received 1.8 Gy per fraction. The organs at risk (OAR) dose constraints were: $50 \%$ of bladder volume must not receive above $50 \mathrm{~Gy}, 35 \%$ of the intestines must not receive dose above $35 \mathrm{~Gy}, 60 \%$ of rectal volume must not receive dose above $50 \mathrm{~Gy}$, and $10 \%$ of the femoral heads must not receive above 50Gy.

All patients received image-guided adaptive brachytherapy (IGABT) dose of 6 Gy in four-fractions (24 Gy) and the total equivalent dose in 2 Gy fraction $\left(\mathrm{EQD}_{2}\right)$ is $32 \mathrm{~Gy}$. The vagina was adequately packed with gauze to keep the rectum and bladder away from receiving a high dose of radiation. Images set obtained by CT- MRI scan was transferred to the Oncentra planning system. microSelectron (Iridium192) was used during the treatment.

\section{Chemotherapy}

Intravenously infused $30 \mathrm{mg} / \mathrm{m}^{2}$ of cisplatin were received by all patients over half an hour and instantly followed by intravenously infused 125 $\mathrm{mg} / \mathrm{m}^{2}$ of gemcitabine about half an hour for 6 weeks thus once per week combined with radiation therapy for 6 weeks from Monday - Friday. Both drugs were given 1 to 2 hours prior to radiation therapy. Patients received adaptive image-guided brachytherapy
(IGABT) on the 7th week immediately after completing chemoradiotherapy plan which, was followed by 14 days of rest. Patients subsequently received two successive 3 weeks' cycle of adjuvant chemotherapy with $1000 \mathrm{mg} / \mathrm{m}^{2}$ of gemcitabine on days 1 and 8 and $25 \mathrm{mg} / \mathrm{m}^{2}$ of cisplatin on day 1-3.

\section{Treatment Evaluation}

Multiple parameters were controlled in accordance with the institutional standards of Zhengzhou university people's hospital and were required to meet the standard safety criteria before the commence of every chemotherapy cycle. Haematology and chemistry results were obtained weekly before starting every cycle of chemotherapy. Chemoradiotherapy was paused when the leucocytes were < $2000 / \mathrm{mm}^{3}$, thrombocytes <100, 000/ $\mathrm{mm}^{3}$, or neurotoxicity below grade 3 per CTCAE v4. Blood transfusion was given to patients with haemoglobin $(\mathrm{Hb})$ level of less than $80 \mathrm{~g} / 1$. Non-haematological and haematological side effects due to chemotherapy were recorded based on the Common Terminology Criteria for Adverse Effects Version 4.0 The Response Evaluation Criteria in Solid Tumours (RECIST v1.1) was used in evaluating the response of the tumour to the treatment.

\section{Statistical Method}

Statistical assessment was conducted using SPSS Version 23.0. The median PFS and median OS were evaluated with the Kaplan - Meier curve. All the data recorded was entered into the excel file and evaluated in proportions and percentages whenever necessary.

\section{Results}

\section{Patients Clinical Characteristics}

The clinical features of the 81 patients involved in our analysis are presented in Table 1 . The median age was 45 (Range: 25-60). Sixty-six patients had the squamous cell carcinoma histological subtype, and fifteen $(18.5 \%)$ had adenocarcinoma subtype. The cervix tumour varied from about $3.5-8.1 \mathrm{~cm}$ at their greatest dimension and the median size was $4.5 \mathrm{~cm}$. Symptoms at diagnosis were vaginal bleeding, vaginal discharge and dyspareunia. Seven patients $(8.6 \%)$ were stage IB2, 10 patients $(12.3 \%), 27$ patients (33.3\%), 13 patients $(16 \%)$ and 24 patients $(29.6 \%)$ were stage IIA, IIB, IIIA and IIIB respectively based on the 2009 FIGO guidelines for staging cervical cancer. Thirty-five (35) patients $(43.2 \%)$ had positive para-aortic lymph node.

\section{Survival Outcomes and treatment efficacy}

Short-term therapy responses were assessed three months after concurrent chemoradiotherapy 
and IGABT. Sixty-nine patients obtained complete response (CR), six had a partial response (PR), and five patients had stable disease (SD). The disease control rate (DCR $=S D$ and ORR) and overall response rate $(\mathrm{ORR}=\mathrm{PR}, \mathrm{CR}$ or $\mathrm{PR})$ were $92.6 \%$ and $85.2 \%$ respectively. The 3-year and 5-year estimated OS was $75.4 \%$ and $66.3 \%$, and the 3-year and 5-year estimated PFS were $78.2 \%$ and $65.4 \%$ (Figure $1 \& 2$ ). The median OS time and PFS time were 45.5 months and 36.8 months, respectively. Distance metastasis was evident in the lung (3 patients), pelvic wall (2 patients), liver (3 patients) and bone (2 patients). Six (6) had a local relapse, and two (2) patients had local relapse plus simultaneous systemic metastatic tumour.

\section{Early and Late Clinical Toxicity}

The toxicities of concurrent chemoradiotherapy were graded by CTCAE v4. No interruption was observed during the treatment. The treatment was completed by all patients and toxicity was well tolerated. Nausea was recorded in $9(8.6 \%)$ patients, 4 patients were grade $1(4.9 \%), 3$ were grade 2 , and 2 patients were grade 3 . Eight patients and 5 patients had grade 1 and grade 2 diarrhoea, respectively. Ten (10) patients had anorexia, and 6 patients had vomiting. (4 were grade 1 , and 2 were grade 2 ). Thrombocytopenia was observed in 8 patients, and 10 patients had anaemia. Seven patients had grade 1 neutropenia. Late genitourinary and gastrointestinal toxicity was noticed in $13(16.0 \%)$ patients. Six $(7.4 \%)$ had grade 1upper gastrointestinal toxicities, 5 patients $(6.2 \%)$ had grade 1 cystitis, and one patient had grade $2(1.2 \%)$ cystitis. Grade 1 dryness of the vagina was evident in $4(4.9 \%)$ patients, two had grade $1(2.5 \%)$ shortening of vagina, and three $(3.7 \%)$ patients had grade 2 vaginal stenosis. Table 2 shows the summary of early and late toxicities.

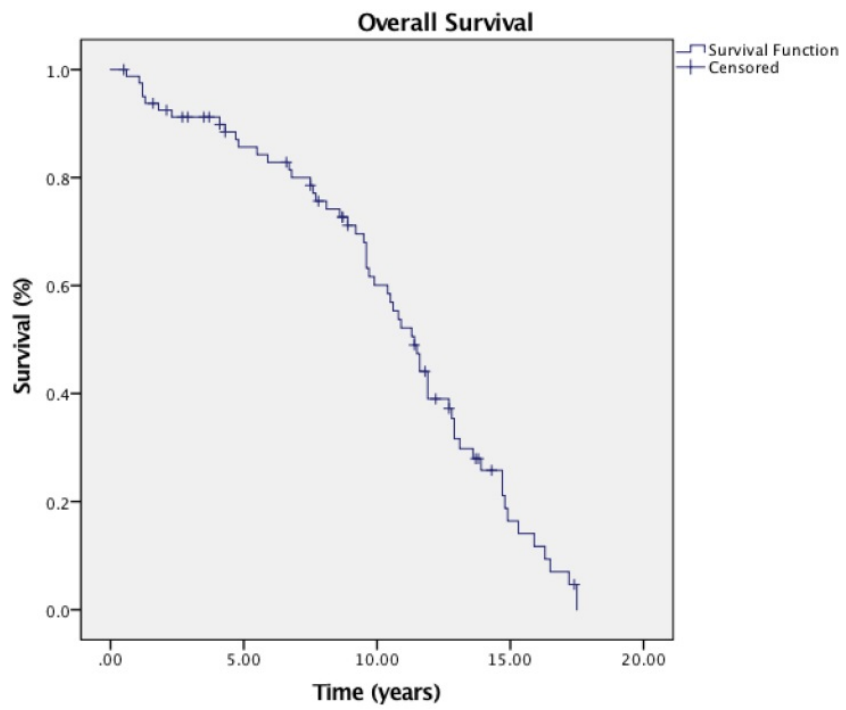

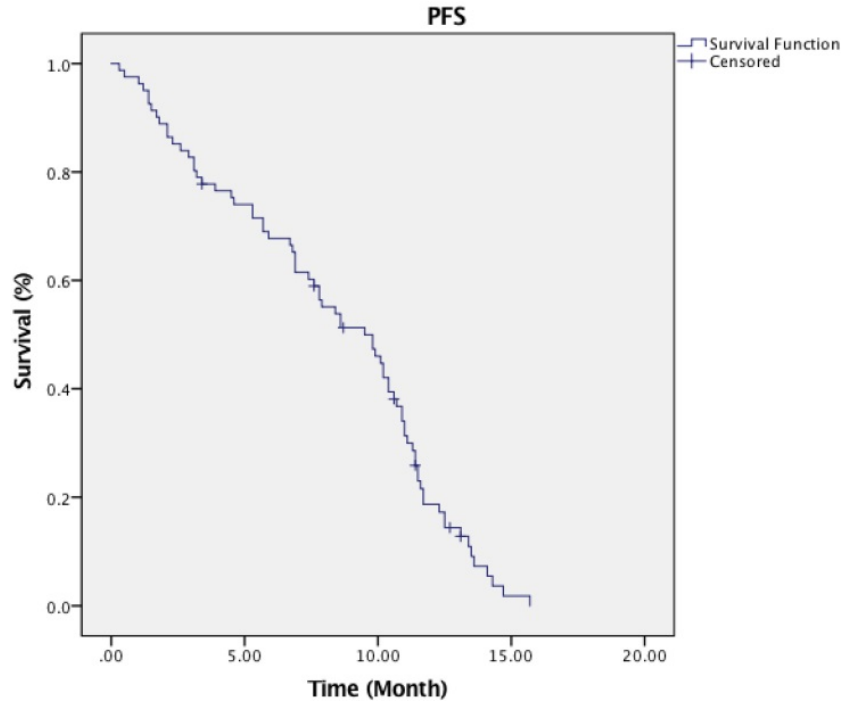

Figure 2. Progression-free survival curve of patients.

Table 1. Patients clinical characteristics.

\begin{tabular}{lll}
\hline Characteristics & NO. Patients & (n \%) \\
\hline Age & & \\
$<55$ & 63 & $77.8 \%$ \\
$\geq 55$ & 18 & $22.2 \%$ \\
Histology type & & \\
SCC & 66 & $81.5 \%$ \\
Adenocarcinoma & 15 & $18.5 \%$ \\
Stage & & \\
IB2 & 7 & $8.6 \%$ \\
IIA & 10 & $12.3 \%$ \\
IIB & 27 & $33.3 \%$ \\
IIIA & 13 & $16.0 \%$ \\
IIIB & 24 & $29.6 \%$ \\
Grade & & \\
1 & 6 & $7.4 \%$ \\
2 & 54 & $66.7 \%$ \\
3 & 21 & $25.9 \%$ \\
Tumour Size & & \\
$<4$ cm & 23 & $28.4 \%$ \\
$\geq 4 \mathrm{~cm}$ & 58 & $71.6 \%$ \\
LNM & & \\
1 & 26 & $32.1 \%$ \\
2 & 21 & $25.9 \%$ \\
$3 \&$ above & 34 & $42.0 \%$ \\
Para-aortic LNM & & \\
Yes & 35 & $43.2 \%$ \\
No & 46 & $56.8 \%$ \\
\hline
\end{tabular}

Table 2. Early and late toxicities during treatment.

\begin{tabular}{lllll}
\hline Toxicity & \multicolumn{2}{l}{ Grade } & & \\
\cline { 2 - 5 } & $\mathbf{1}$ & $\mathbf{2}$ & $\mathbf{3}$ & $\mathbf{4}$ \\
\hline Anaemia & $8(9.9 \%)$ & $2(2.5 \%)$ & 0 & 0 \\
Neutropenia & $6(7.4 \%)$ & $1(1.2 \%)$ & 0 & 0 \\
Thrombocytopenia & $5(6.2 \%)$ & $3(1.4 \%)$ & 0 & 0 \\
Nausea & $4(4.9 \%)$ & $3(7.1 \%)$ & $2(2.5 \%)$ & 0 \\
Vomiting & $4(4.9 \%)$ & $2(2.5 \%)$ & 0 & 0 \\
Diarrhea & $8(9.9 \%)$ & $5(6.2 \%)$ & 0 & 0 \\
Anorexia & $8(9.9 \%)$ & $2(2.5 \%)$ & 0 & 0 \\
Late gastrointestinal & $7(8.6 \%)$ & 0 & 0 & 0 \\
Late genitourinary & $5(6.2 \%)$ & $1(1.2 \%)$ & 0 & 0 \\
\hline
\end{tabular}




\section{Discussion}

Radiotherapy alone has unsatisfying outcomes in the management and treatment of locoregionally advanced carcinoma of the cervix. Several studies have been designed and undertaken over the last decade with the objective of improving treatment result. In a meta-analysis performed by Vale $\mathrm{C}$ et al on individual patient information, the combined findings of these crucial studies were assessed [10]. The inclusion of chemotherapy decreased both distant and local recurrence and contributed to about a $6 \%$ increase in 5-year survival when compared to only radiotherapy [15]. Chemoradiotherapy with cisplatin was recognized as the standard care of treatment after the National Cancer Institute notice in 1999. Cisplatin is well tolerated on a weekly basis and demonstrates better outcomes in research compared to radiation therapy alone. Nevertheless, the cure rate remains low, and the 5-year response rate is about $60 \%$ [16].

In concurrent chemoradiotherapy protocols, non-platinum compounds alone were rarely evaluated and were usually overlooked in the literature. In a few studies, 5-FU (fluorouracil) and cisplatin were tested, but the problem was toxicity, and the dimension of frequent treatment gaps was also highlighted. Four out of the five studies in which cisplatin was accepted to be the standard treatment had 5-FU combined with cisplatin [16-17]. Equally, gemcitabine remains another underused chemotherapeutic agent in concomitant chemoradiotherapy setting of cervical cancers. Compared to the conventional concurrent cisplatin routine in cervical cancer, we do not have much information on its effectiveness due to the absence of studies.

Gemcitabine is part of the group of antimetabolites (2', 2'-di-fluorodeoxycytidine) that inhibits the synthesis of DNA and ultimately causes programmed cell death. Gemcitabine has demonstrated effectiveness against a multitude of tumours, particularly lung and pancreatic cancer. It was also evaluated in gastrointestinal (GI) cancers, breast, urinary bladder, ovary and cancer of the cervix [18]. Gemcitabine was originally tested for moderate activity in recurrent and metastatic cervical cancers. The findings were comparatively small when used alone in postradiation or recurrence residual tumours. Gemcitabine is rarely used alone in treating cervix carcinoma in a residual/recurrence setting owing to less than anticipated outcomes [16,18].

Studies in vivo and in vitro have shown that gemcitabine is a powerful radiosensitizer that dramatically increases the outcomes when coupled with radiotherapy $[17,19]$. It also demonstrates synergistic action with cisplatin, the likely cause being
DNA cisplatin adducts repair suppression by gemcitabine [19].

Burnett et al. reported a total response rate of $41 \%$ with a reasonable toxicity effect [20]. The neoadjuvant application of gemcitabine plus cisplatin chemotherapy was investigated by González et al [21-22]. They reported that the most prevalent haematological toxicity was grade 3 granulocytopenia in $13.8 \%$ of the patients and grade 4 granulocytopenia in $3.4 \%$ of the patients. The further evaluation also demonstrated that the neoadjuvant strategy is at least as efficient as standard concomitant chemoradiotherapy involving cisplatin.

A phase I and II trial conducted by Zarba et al investigated weekly gemcitabine and cisplatin in thirty-six patients. They discovered that a peak acceptable dose of $150 \mathrm{mg} / \mathrm{m}^{2}$ is the peak tolerability dose for gemcitabine when used weekly during concurrent chemoradiotherapy [23]. In addition, Umanzor et al. reported well-tolerated toxicity in 23 patients treated with concurrent gemcitabine plus cisplatin. In the same study, grade 3 neutropenia was evident in one patient. Secondly, no haematological toxicity of grade 4 and no unusual toxicity of late radiation was observed [24]. A pivotal study conducted by González et al endorsed those early studies. The researchers compared concurrent chemoradiotherapy involving cisplatin and gemcitabine followed by adjuvant cisplatin and gemcitabine in locally advanced carcinoma of the cervix. Their results showed an improved 3-year OS [HR:0.68, 95\% CI: 0.49-0.95, p-value $=0.022$ ]. Similar improvement was achieved in 3-year PFS $[74.4 \%$ vs $65.0 \%$, $\mathrm{p}$-value $=0.029]$ and lower distant metastasis rate [ $8.1 \%$ vs $16.4 \%$, p-value $=0.005]$. Nevertheless, grade 3 and 4 toxicity was coupled with this success $[86.5 \%$ vs. $46.3 \%$, p-value $=0.001]$ [7].

Compared to prior studies, our research has several unique methodological and patient characteristics. First, we used highly conformal radiotherapy in treating our patients while conventional external beam radiotherapy was used in previous studies. This is the possible reason for the lower rate of haematological toxicity achieved in our studies. In addition, IMRT has been shown to be superior to three-dimensional (3D) and two-dimensional (2D) methods, both in terms of reducing toxicity incidence and bone-marrow sparing [25-26].

The findings of our study are similar to those reported in the literature. Loco-regional control is superior to past studies that used the gemcitabine plus cisplatin regimen. This can be ascribed to the use of intrauterine adaptive brachytherapy other than chemotherapy inclusion. Local failure was a problem in González et al study, though there was no 
statistical difference when the rate of local failure was considered ( $16.4 \%$ vs. $11.2 \%, p=0.097)$. Nevertheless, tumour control is challenging since systemic metastasis is reported in about $40 \%$ of patients [7].

Modern radiation therapy technique has resulted in outstanding and excellent local control rates. Regardless of tumour size the 3-year local control rate goes beyond $95 \%$ and approaches approximately $100 \%$ if the equivalent total dose (EQD2) to the volume of the "high-risk tumour" is higher than $87 \mathrm{~Gy}$ [27]. These observations lead to the hypothesis that the combinations of chemotherapy during radiotherapy have an important impact. Moreover, even without visible local tumour at the moment of chemoradiotherapy, the microscopic tumour could still be harboured in the local lymph nodes, and the macroscopic tumour may likewise be available and stay unnoticed by radiological examinations like MRI or computed tomography [28-29]. It is likely that giving chemotherapy during external beam radiotherapy may contribute to the effective eradication of tumours in the lymph nodes.

Small sample size, short-follow up, and the retrospective nature of the study are some of the limitations in our study. Notwithstanding these restrictions, we believe our research offers a distinctive perspective into new therapeutic options for patients with locoregionally advanced carcinoma of the cervix. The data presented can serve as a foundational guide for future randomized controlled trials.

\section{Abbreviations}

CCRT: concomitant chemoradiotherapy; LRACC: loco-regionally advanced carcinoma of the cervix; PTV: Planning target volume; CTV: Clinically target volume; RTOG: Radiation Therapy Oncology Group; Gy: Gray.

\section{Acknowledgements}

We would like to express our gratitude to all the doctors, nurses and medical physicists of the Zhengzhou University People's Hospital and Henan Provincial People's Hospital.

\section{Funding}

This study was partially supported by the National Natural Science Foundation of China (No. 81971508, No. 81471589, No. 81273259), the Health Bureau of Henan Province, P.R. China (No. 201201005) and the foundation and frontier research grant of Henan provincial science and technology bureau, PR China (No.112300410027, No. 132102310120).

\section{Availability of materials}

The data analysed for the current study is available with the corresponding author and can be released on reasonable request.

\section{Ethics approval and consent to participate}

The ethics board of Zhengzhou University and Henan Provincial People's Hospital approved this study.

\section{Competing Interests}

The authors have declared that no competing interest exists.

\section{References}

1. Torre LA, Bray F, Siegel RL, Ferlay J, Lortet-Tieulent J, Jemal A. Global cancer statistics 2012. CA Cancer J Clin. 2015; 65: 87-108.

2. Nedovic J, Protrka Z, Ninkovic S, Mitrovic S, Vojinovic R, Glisic J, Markovic-Filipovic B, Milosevic B, Peulic M, Cvetkovic A. Cisplatin monotherapy with concurrent radiotherapy versus combination of cisplatin and 5-fluorouracil chemotherapy with concurrent radiotherapy in patients with locoregionally advanced cervical carcinoma. J BUON. 2012; 17: 740-745.

3. Park B, Kim SI, Seo SS, Kang S, Park SY, Lim MC. Health Behaviors and Associated Sociodemographic Factors in Cervical Cancer Survivors Compared with Matched Non- Cancer Controls. PLoS One. 2016; 11: e0160682.

4. Lee CH, Chang JS, Syu SH, Wong TS, Chan JY, Tang YC, Yang ZP, Yang WC, Chen CT, Lu SC, Tang PH, Yang TC, Chu PY, et al. IL-1beta promotes malignant transformation and tumor aggressiveness in oral cancer. J Cell Physiol. 2015; 230: 875-884.

5. Liu H, Antony S, Roy K, Juhasz A, Wu Y, Lu J, Meitzler JL, Jiang G, Polley E, Doroshow JH. Interleukin-4 and interleukin-13 increase NADPH oxidase 1-related proliferation of human colon cancer cells. Oncotarget. 2017; 8: 3811335 .

6. Kidd EA, Siegel BA, Dehdashti F, Rader JS, Mutic S, Mutch DG, Powell MA, Grigsby PW. Clinical outcomes of definitive intensity-modulated radiation therapy with fluorodeoxyglucose-positron emission tomography simulation in patients with locally advanced cervical cancer. Int J Radiat Oncol Biol Phys. 2010; 77(4): 1085-91.

7. Duenas-Gonzalez A, Zarba JJ, Patel F, Alcedo JC, Beslija S, Casanova L, Pattaranutaporn P, Hameed S, Blair JM, Barraclough H, et al. Phase III, open label, randomized study comparing concurrent gemcitabine plus cisplatin and radiation followed by adjuvant gemcitabine and cisplatin versus concurrent cisplatin and radiation in patients with stage IIB to IVA carcinoma of the cervix. J Clin Oncol. 2011; 29(13): 1678-85.

8. Lin, J. C. et al. Phase III study of concurrent chemoradiotherapy versus radiotherapy alone for advanced nasopharyngeal carcinoma: positive effect on overall and progression-free survival. J Clin Oncol. 2003; 21: 631-637.

9. Wu LR, et al. Ten-year survival outcomes for patients with nasopharyngeal carcinoma receiving intensity-modulated radiotherapy: An analysis of 614 patients from a single center. Oral oncology. 2017; 69: 26-32.

10. Blanchard, P. et al. Chemotherapy and radiotherapy in nasopharyngeal carcinoma: an update of the MAC-NPC meta-analysis. Lancet Oncology. 2015; 16: 645-655.

11. Hashemi FA, Akbari EH, Kalaghchi B, Esmati E. Concurrent chemoradiation with weekly gemcitabine and cisplatin for locally advanced cervical cancer. Asian Pac J Cancer Prev. 2013; 14: 5385-5389.

12. Cihoric N, Tapia C, Kruger K, Aebersold DM, Klaeser B, Lossl K. IMRT with (1)(8) FDG-PET \CT based simultaneous integrated boost for treatment of nodal positive cervical cancer. Radiat Oncol. 2014; 9: 83.

13. Cihoric N, Tsikkinis A, Tapia C, Aebersold DM, Zlobec I, Lossl K. Dose escalated intensity modulated radiotherapy in the treatment of cervical cancer. Radiat Oncol. 2015; 10: 240.

14. Beadle BM, Jhingran A, Salehpour M, Sam M, Iyer RB, Eifel PJ. Cervix regression and motion during the course of external beam chemoradiation for cervical cancer. Int J Radiat Oncol Biol Phys. 2009; 73(1): 235-41.

15. Vale C, Tierney JF, Stewart LA, et al. Reducing uncertainties about the effects of chemoradiotherapy for cervical cancer: a systematic review and meta-analysis of individual patient data from 18 randomized trials. J Clin Oncol. 2008; 26(35): 5802-12.

16. Chen M, Hough AM, Lawrence TS. The role of p53 in gemcitabine mediated cytotoxicity and radiosensitization. Cancer Chemother Pharmacol. 2000; 45(5): 369-74.

17. Murphy JD, Lucas DR, Somnay YR, Hamstra DA, Ray ME. Gemcitabine mediated radiosensitization of human soft tissue sarcoma. Transl Oncol. 2008; 1(1): 50-6. 
18. Zhen $\mathrm{Z}$, Ruo-Nan $\mathrm{Y}, \mathrm{Li} \mathrm{T}$, et al. Assessment of Concurrent Chemoradiotherapy plus Induction Chemotherapy in Advanced Nasopharyngeal Carcinoma: Cisplatin, Fluorouracil, and Docetaxel versus Gemcitabine and Cisplatin. Scientific Reports. 2018; 8: 15581.

19. Cihoric N, Tsikkinis A, Tapia C, Aebersold DM, Zlobec I, Lossl K. Highly conformal combined radiotherapy with cisplatin and gemcitabine for treatment of loco-regionally advanced cervical cancer - a retrospective study. Radiation Oncology. 2017; 12: 202.

20. Burnett AF, Roman LD, Garcia AA, Muderspach LI, Brader KR, Morrow CP. A phase II study of gemcitabine and cisplatin in patients with advanced, persistent, or recurrent squamous cell carcinoma of the cervix. Gynecol Oncol. 2000; 76(1): 63-6.

21. Dueñas-Gonzalez A, Lopez-Graniel C, Gonzalez A, Reyes M, Mota A, Muñoz D, Solorza G, Hinojosa LM, Guadarrama R, Florentino R, et al. A phase II study of gemcitabine and cisplatin combination as induction chemotherapy for untreated locally advanced cervical carcinoma. Ann Oncol. 2001; 12(4): 541-7.

22. Duenas-Gonzalez A, Lopez-Graniel C, Gonzalez-Enciso A, Mohar A, Rivera L, Mota A, Guadarrama R, Chanona G, De La Garza J. Concomitant chemoradiation versus neoadjuvant chemotherapy in locally advanced cervical carcinoma: results from two consecutive phase II studies. Ann Oncol. 2002; 13(8): 1212-9.

23. Zarba JJ, Jaremtchuk AV, Gonzalez Jazey P, Keropian M, Castagnino R, Mina C, Arroyo G, Argentina G. A phase I-II study of weekly cisplatin and gemcitabine with concurrent radiotherapy in locally advanced cervical carcinoma. Ann Oncol. 2003; 14(8): 1285-90.

24. Umanzor J, Aguiluz M, Pineda C, Andrade S, Erazo M, Flores C, Santillana S. Concurrent cisplatin/gemcitabine chemotherapy along with radiotherapy in locally advanced cervical carcinoma: a phase II trial. Gynecol Oncol. 2006; 100(1): 70-5.

25. Erpolat OP, Alco G, Caglar HB, Igdem S, Saran A, Dagoglu N, Aslay I, Ozsaran Z, Demirci S, Keven E, et al. Comparison of hematologic toxicity between 3DCRT and IMRT planning in cervical cancer patients after concurrent chemoradiotherapy: a national multi-center study. Eur J Gynaecol Oncol. 2014; 35(1): 62-6.

26. Chuong MD, Freilich JM, Hoffe SE, Fulp W, Weber JM, Almhanna K, Dinwoodie W, Rao N, Meredith KL, Shridhar R. Intensity-modulated radiation therapy vs. 3D conformal radiation therapy for Squamous cell carcinoma of the Anal Canal. Gastrointest Cancer Res. 2013; 6(2): 39-45.

27. Dimopoulos JC, Lang S, Kirisits C, Fidarova EF, Berger D, Georg P, Dorr W, Potter R. Dose-volume histogram parameters and local tumor control in magnetic resonance image-guided cervical cancer brachytherapy. Int J Radiat Oncol Biol Phys. 2009; 75(1): 56-63.

28. Grigsby PW, Siegel BA, Dehdashti F. Lymph node staging by positron emission tomography in patients with carcinoma of the cervix. J Clin Oncol. 2001; 19(17): 3745-9.

29. Kidd EA, Siegel BA, Dehdashti F, Rader JS, Mutch DG, Powell MA, Grigsby PW. Lymph node staging by positron emission tomography in cervical cancer: relationship to prognosis. J Clin Oncol. 2010; 28(12): 2108-13. 